\title{
Mejor no saber \\ Sobre la doctrina de la ignorancia deliberada en Derecho penal
}

Ramon Ragués i Vallès

Universitat Pompeu Fabra - Barcelona

\section{La evitación del conocimiento como decisión cotidiana y su trascendencia jurídica}

Un estudiante sabe que las notas del último examen se publicarán el viernes pero prefiere no consultarlas hasta el lunes para no echar a perder el fin de semana si los resultados son malos; un cónyuge sospecha que el otro le es infiel, pero decide no investigar por temor a confirmar sus sospechas; un alpinista debe caminar en su ascensión junto a un profundo precipicio y prefiere no mirar al lado por miedo a no tener valor suficiente para cruzar... Todos estos casos -en principio jurídicopenalmente irrelevantes- son ejemplos de ignorancia deliberada: describen situaciones en las que un sujeto podía haber obtenido determinada información pero, por razones muy diversas, ha preferido no adquirirla y mantenerse en un estado de incertidumbre. Se trata de una estrategia, por así decirlo, humanamente comprensible para quien desea evitar o aplazar un posible disgusto o tener que tomar una decisión difícil.

Los casos de ignorancia deliberada también pueden presentarse en hechos con trascendencia jurídico-penal: imagínese, por ejemplo, el caso del testaferro profesional que, a cambio de una retribución, acepta figurar como administrador formal de cientos de sociedades de las que no le importa lo más mínimo la actividad real o quiénes son sus verdaderos gestores; o el sujeto que, para evitarse problemas, da instrucciones a sus empleados de que rechacen la entrega de cualquier 
tipo de notificación futura que pueda provenir de la administración; o, por fin, de quien recibe una importante cantidad de dinero a cambio de transportar una maleta a otra ciudad y prefiere no preguntar exactamente qué es lo que se aloja en el interior.

En el Derecho penal moderno la atribución de responsabilidad por la realización de una gran mayoría de comportamientos penalmente prohibidos requiere que el sujeto sea conocedor de aquellas características objetivas de su hecho que lo convierten en penalmente típico. Dicho a la inversa, el conocimiento insuficiente o el error son causas de exención de responsabilidad, salvo en aquellos casos excepcionales en los que se prevé expresamente el castigo de las conductas realizadas sin dicho conocimiento, como sucede en los delitos imprudentes $\mathrm{o}$, en los sistemas angloamericanos, en las infracciones de strict liability. ¿Cómo debe enfrentarse a los casos de ignorancia deliberada un Derecho penal que considera el conocimiento como un elemento básico de la responsabilidad? ¿Resulta aceptable que un sujeto que ha buscado permanecer en el desconocimiento se beneficie penalmente de tal circunstancia? ¿Tiene sentido que aquellos que se esfuerzan por conocer sean de peor condición para el Derecho penal que quienes evitan profundizar en los detalles de sus actuaciones?

El presente trabajo se propone analizar las posibles respuestas a las anteriores cuestiones, que obligan a repensar algunos de los aspectos básicos de lo que suele denominarse la responsabilidad subjetiva o la mens rea en los sistemas angloamericanos. Para ello, se dedicarán dos primeros apartados (II y III) a exponer cómo los dos grandes sistemas-jurídico penales -el Common Law y el Derecho continental- se enfrentan a los supuestos de evitación intencionada del conocimiento. Y, tras un análisis crítico de los problemas no resueltos (IV), se propondrán algunos criterios para lo que se considera que podría ser un tratamiento más adecuado de estas situaciones (V). Anticipando una conclusión, se pondrá en evidencia cómo algunos casos de ignorancia deliberada -aqui denominados de ignorancia "en sentido estricto"- son una pequeña grieta que en el sistema continental de imputación subjetiva amenaza la solidez de las bases de dicho sistema. 
Mejor no saber

\section{La ignorancia deliberada en el Common Law: la doctrina de la willful blindness}

La primera vez que en el common law se sostuvo la necesidad de equiparar penalmente el efectivo conocimiento y los casos de "ceguera intencionada" (willful blindness) fue en la sentencia inglesa de 1861 sobre el caso Regina v. Sleep ${ }^{1}$. El jurado que enjuició en primera instancia a Mr. Sleep le condenó como autor de un delito de malversación por haberse apropiado de tornillos de cobre marcados como de titularidad pública. Ante su alegación afirmando no haber sido consciente de que los bienes eran públicos, el juez Willes concluyó que la condena debía revocarse porque "el jurado no ha[bía] considerado acreditado que el individuo conociera que los bienes estaban marcados [como propiedad estatal] ni tampoco que se abstuviera intencionadamente de adquirir tal conocimiento" 2 . Según parece desprenderse de este pasaje -ciertamente poco elocuente- la abstención intencionada de obtener conocimientos, en caso de haberse probado, habría debido merecer la misma respuesta punitiva que el conocimiento efectivo.

En 1899 la doctrina apareció por vez primera en una resolución del Tribunal Supremo de los Estados Unidos, concretamente en la sentencia del caso Spurr v. United States. En ella se revisaba la condena de $M r$. Spurr, culpable de haber certificado los cheques emitidos por un cliente contra una cuenta que carecía de fondos. De acuerdo con la ley aplicable, para sancionar penalmente tal conducta era necesaria una violación intencionada de los preceptos que regulaban la emisión de dichos efectos mercantiles. En su recurso la defensa de Spurr cuestionó que el jurado hubiera sido correctamente instruido, pues el magistrado que lo presidía no informó de que el delito aplicable exigía que el acusado actuase "intencionadamente", es decir, que la conducta de quien actuaba en la creencia errónea de que existían fondos en la

1 Robbins, JCLC, 81 (1990), p. 196; Marcus, YLJ, 102 (1993), pp. 2233-2234; y EDWARDS, $M L R, 17$ (1954), p. 298 ss. Una perspectiva sobre los orígenes y el desarrollo de esta doctrina en RAGUÉS I VALLĖs, La ignorancia, capítulo 2.

2 Cita tomada de RobBins, JCLC, 81 (1990), p. 196, que aporta las referencias originales. 
cuenta en el momento de emitir la certificación no era penalmente relevante. En respuesta a semejante alegación, el Tribunal Supremo formuló obiter dictum las siguientes apreciaciones:

"El propósito infractor es la esencia del presente delito. Si el oficial certifica un cheque con la intención de que el emisor obtenga dinero del banco pese a no tener fondos, dicha certificación no solo es ilícita, sino que se le puede atribuir el propósito específico de violar la ley. Y este mal propósito puede presumirse cuando el oficial se mantiene deliberadamente en la ignorancia acerca de si el librador tiene o no dinero en el banco o cuando muestra una indiferencia crasa (grossly indifferent) respecto de su deber de asegurarse de tal circunstancia" ${ }^{3}$.

El Tribunal Supremo acabaría estimando el recurso de Spurr coincidiendo con su defensa en que el jurado no había sido correctamente informado acerca de la exigencia de intención por parte del delito aplicable. Aunque en semejante decisión el anterior pasaje no tendría ninguna trascendencia real, lo cierto es que en los siguientes años las frases reproducidas serían habitualmente citadas por numerosos tribunales, equiparando el desconocimiento provocado con el conocimiento efectivo. Sin embargo, el número de resoluciones en las que aparece la willful blindness no sería especialmente elevado hasta la década de 1970, cuando esta doctrina empezaría a aplicarse de manera generalizada en casos de transporte de droga ${ }^{4}$. En este último contexto se enmarca, por ejemplo, la sentencia del caso United States v. Jewell ${ }^{5}$, que suele citarse como el leading case en la materia. El acusado Jewell había sido condenado en primera instancia por cruzar la frontera de México con los Estados Unidos transportando, supuestamente por encargo, 110 libras de marihuana en el compartimento secreto del maletero de

3174 U.S. 728 (1899), p. 735. Sobre este caso RobBIns, JCLC, 81 (1990), pp. 197-198; CHARLOW, TLR, 70 (1992), pp. 1353-1354 (nota 7) y 1359; y Marcus, YLJ, 102 (1993), pp. 2234-2235.

${ }^{4}$ Cfr. el comentario al caso United States v. Murrieta-Bejarano publicado en la Iowa Law Review, 63 (1977/78), pp. 470-471.

5532 F. 2d 697 (9th Cir. 1976). Al respecto ver también RoBBins, JCLC, 81 (1990), p. 203 ss; Charlow, TLR, 70 (1992), p. 1419; y Marcus, YLJ, 102 (1993), pp. 2241-2243. 
un coche. Su alegación exculpatoria de que no sabía exactamente lo que transportaba, pese a tener la sospecha de estar haciendo algo ilegal, fue desestimada por un jurado instruido en los siguientes términos:

La acusación satisface la carga de la prueba demostrando, más allá de toda duda razonable, que si el acusado no era en realidad consciente de que había marihuana en su vehículo cuando entró en los Estados Unidos fue porque su desconocimiento acerca de esta circunstancia fue única y exclusivamente el resultado de haberse hecho el propósito consciente de ignorar la naturaleza de lo que llevaba en el coche, con una voluntad consciente de evitar conocer la verdad.

La condena del jurado fue recurrida por Jewell, cuya defensa cuestionó la legalidad de esta instrucción. Sin embargo, en su posterior resolución el Tribunal de Apelaciones confirmaría la condena partiendo de la equiparación que contiene la sección 2.02.7 del Model Penal Code entre conocimiento cierto de un determinado hecho y conciencia de la alta probabilidad de su concurrencia. Según la Sala, quien es consciente de la alta probabilidad de la existencia de un hecho y no hace lo necesario para confirmar su sospecha merece el mismo tratamiento que quien tiene plena certeza sobre tal extremo. Para justificar su decisión el Tribunal invocó, entre otras, la idea de que "la ignorancia deliberada y el conocimiento positivo presentan un mismo grado de culpabilidad"6.

Con todo, y precisamente a raíz de la publicación del Model Penal Code -un texto no legislativo pero que ha servido de pauta para la reforma de muchos códigos penales estadounidenses- en la discusión académica de las últimas décadas se viene cuestionando de manera creciente la actual vigencia de la willful blindness, que no fue incluida expresamente entre las formas de imputación subjetiva que aparecen en la sección 2.02 de este texto. Ello ha llevado a algunos autores a entender que las situaciones abarcadas por esta forma de imputación deberían reconducirse a la recklessness (figura muy similar al dolo even-

${ }^{6}$ Robbins, JCLC, 81 (1990), p. 205 y Husak/Callender, WLR, 29 (1994), p. 35. 
tual continental), mientras otros académicos denuncian que con esta doctrina se estarían rebasando los límites impuestos por el principio de legalidad ${ }^{7}$. Sea como fuere, y pese a semejantes dudas, la teoría no ha dejado de aplicarse por los tribunales y ha ampliado incluso su radio de acción a ámbitos como la criminalidad medioambiental y corporativa en general ${ }^{8}$.

Así, a mediados de 2011 la vigencia de la doctrina se ha visto confirmada de nuevo por el propio Tribunal Supremo en su sentencia sobre el caso Global-Tech Appliances, Inc. et al. v. Seb S.A., a propósito de un procedimiento civil por infracción del derecho de patente ${ }^{9}$. En esta resolución el Tribunal afirma que "la doctrina de la ignorancia deliberada está bien asentada en Derecho penal" y entiende que no existe ninguna objeción en emplearla también en asuntos civiles a propósito de normas que exijan para su infracción un determinado conocimiento. Sin embargo, al precisar el alcance de la doctrina entiende la Corte, en la línea de la Sección 2.02.7 del Código Penal Modelo, que esta teoría exige que el acusado haya obrado siendo consciente de la alta probabilidad de la concurrencia de un determinado hecho, una exigencia que restringe notablemente su ámbito de aplicación y que, por más que el Tribunal sostenga expresamente lo contrario, dificulta su distinción respecto de la recklessness, que concurre cuando el sujeto "desprecia conscientemente un riesgo sustancial e injustificado de que concurra un determinado elemento material o éste acabe resultando de su conducta" (sección 2.02.c del Código Modelo).

7 Sobre el sistema de imputación subjetiva previsto en el Código Penal Modelo y la polémica generada acerca de la vigencia de la doctrina de la willful blindness cfr. RAGUÉS I VALLÈs, La ignorancia, pp. 69-92, con ulteriores referencias bibliográficas.

${ }^{8} \mathrm{El}$ caso de referencia a propósito de la criminalidad ecológica es United States v. MacDonald \& Watson Waste Oil Co., resuelto por el Tribunal de Apelaciones del Primer Circuito en 1991 (933 F. 2d 35 [1st Cir. 1991]).

9563 U.S. _ (2011). 


\section{E1 desconocimiento provocado en el Derecho continental}

En los sistemas continentales de influencia germánica -como la mayoría de los latinoamericanos o el español- supuestos como los descritos, en los que una persona renuncia deliberadamente a conocer (o a conocer mejor) determinadas circunstancias de su conducta, suelen reconducirse a aquella modalidad de dolo denominada "dolo eventual" pues, pese a su renuncia a conocer, en tales casos el sujeto cuenta ya con un conocimiento básico suficiente para atribuirle tal forma de dolo ${ }^{10}$ : así, por ejemplo, en el caso del transporte de la maleta con droga bastará con que el acusado haya sido consciente de la posibilidad de estar transportando dicha sustancia para entender que ha obrado dolosamente, aun cuando no haya llegado a cerciorarse plenamente de todas las características concretas (naturaleza, peso, pureza) del objeto del delito.

El amplio alcance del dolo como modalidad de imputación subjetiva, que abarca desde los casos de auténtica intención hasta aquellos supuestos en los que el sujeto simplemente se representa el riesgo de realización típica, permite evitar que la gran mayoría de tales supuestos permanezca impune. Ciertamente para el dolo una parte muy importante de la doctrina exige también la concurrencia de un elemento supuestamente volitivo (que suele caracterizarse con expresiones tales como "consentimiento", "aprobación" o "conformidad"), pero en los casos de dolo eventual la concurrencia de tal elemento suele presumirse cuando el sujeto ha obrado pese a contar con la representación de un nivel importante de riesgo ${ }^{11}$. El hecho de que la gran mayoría de códigos penales no contenga una definición de dolo facilita, sin duda, que la jurisprudencia y la doctrina ajusten los contornos de esta figura según la respuesta punitiva que consideran adecuada para cada caso o grupo de casos.

${ }^{10}$ En tal sentido los argumentos ya expuestos en RAGUÉs I VALLĖs, La ignorancia, p. 101 ss.

${ }^{11} \mathrm{Al}$ respecto FRISch, Vorsatz, p. 381 ss, entre otros, ha puesto en evidencia cómo, especialmente en relación con las circunstancias típicas preexistentes, la jurisprudencia suele conformarse con que el sujeto se represente la posibilidad de su concurrencia, sin que la voluntad desempeñe papel alguno. 


\section{Problemas no resueltos de la ignorancia deliberada}

Sin embargo, la inclusión de los supuestos de mera representación de la posibilidad en el concepto de dolo plantea dos grandes problemas: en primer lugar, el que se cobijen bajo un mismo título de imputación subjetiva -y, en principio, se castiguen con la misma pena-supuestos muy distintos desde el punto de vista del grado de implicación subjetiva del sujeto en el hecho: así, para el planteamiento dominante parece resultar igual de grave el comportamiento de quien transporta droga siendo perfectamente consciente de ello que el de quien solo se plantea como posible tal circunstancia, del mismo modo que se trata igual a quien mata o hiere a otro con intención de hacerlo que a quien realiza un comportamiento peligroso para la integridad o vida ajenas siendo consciente del riesgo pero sin desear el resultado lesivo. Y, como segundo problema, no queda clara la solución que se propone para aquellos supuestos en los que, de manera intencionada, el sujeto haya evitado adquirir incluso aquellos conocimientos mínimos que requiere el dolo eventual, es decir, los supuestos que pueden denominarse como de "ignorancia deliberada stricto sensu". Aunque tales casos no sean tan frecuentes como los de quien albergando sospechas iniciales (suficientes para el dolo eventual) prefiere no profundizar en ellas, no puede de ningún modo descartarse que se presenten en la práctica.

Un ejemplo en tal sentido sería el caso del individuo que diera instrucciones a sus empleados de rechazar cualquier tipo de comunicación oficial proveniente de la administración: ¿podría decirse que concurre el conocimiento requerido por el dolo de un delito de desobediencia si, a resultas de esta orden, años más tarde dicho sujeto no llegara a tener conocimiento de que un juzgado le estaba requiriendo determinada documentación? Una situación similar, por poner otro ejemplo, se plantearía en el caso de la empresa que, como sucede cada vez con mayor frecuencia, se dotara de un buzón electrónico para recibir denuncias de los trabajadores por posibles irregularidades de otros empleados. Imagínese que los directivos decidieran no abrir nunca dicho buzón para evitarse complicaciones y 
que, al cabo de unos años, llegara la denuncia de un trabajador informando de que compañeros suyos incurren de manera permanente en conductas delictivas ipuede afirmarse en tal caso la concurrencia de dolo en los directivos que han ignorado el mensaje y que, por tanto, no han hecho nada para impedir actuaciones ilícitas de sus subordinados? Y la misma situación se daría también en el caso del testaferro que aceptase figurar como administrador formal de cientos de sociedades de las que nada conoce si mucho tiempo más tarde sabe que una de ellas ha sido empleada por su administrador de hecho para cometer un delito de comisión muy poco frecuente.

El planteamiento mayoritario en Derecho continental considera que la falta de conocimiento -siquiera eventual- de la concurrencia en un determinado comportamiento de los elementos de una figura delictiva impide considerar el hecho como doloso, lo que lleva a la impunidad o, cuando la ley lo permita, a castigar la conducta como imprudente. Sin embargo, en casos como los expuestos, en los que el sujeto ha decidido permanecer en la ignorancia, tal conclusión parece insatisfactoria y no deja de resultar extraño afirmar que quien ha evitado conocer está en una situación de error o ha actuado de manera negligente. Seguramente el principal ejemplo de ello sea el Tribunal Supremo español que, de manera ciertamente un tanto errática, ha incorporado a su doctrina la teoría angloamericana de la willful blindness como una modalidad más de dolo.

\section{IV.1. La doctrina del Tribunal Supremo español}

La primera resolución de la Sala Segunda del Tribunal Supremo en la que se mencionó la ignorancia deliberada fue la Sentencia de 10-122000 (ponente Giménez García). En este pronunciamiento se dio respuesta a la alegación de un sujeto, condenado como autor de receptación por haber transportado importantes cantidades de dinero en efectivo a un paraíso fiscal, que afirmó en su descargo no haber sido consciente de que tales cantidades tenían su origen, como así era, en el tráfico de drogas. La Sala rebatió dicha alegación por medio de los siguientes argumentos, que serán reiterados con frecuencia en resoluciones posteriores: 
En la entrega del dinero a José J., Miguel estuvo acompañado de Hebe, y José J. cobraba un 4 \% de comisión. La Sala extrae la conclusión de que José J. tuvo conocimiento de que el dinero procedía del negocio de drogas -cosa que él niega- de hechos tan obvios como que la cantidad era muy importante y de la naturaleza claramente clandestina de las operaciones, por lo que quien se pone en situación de ignorancia deliberada, es decir no querer saber aquello que puede y debe conocerse, y sin embargo se beneficia de esta situación -cobraba un $4 \%$ de comisión-, está asumiendo y aceptando todas las posibilidades del origen del negocio en el que participa, y por tanto debe responder de sus consecuencias (negrita en el original).

Aunque este pasaje no destaca precisamente por su claridad, en él cabe distinguir dos grupos de argumentos que se corresponden, respectivamente, con el elemento cognitivo y volitivo que, según la jurisprudencia tradicional, son necesarios para poder sostener que alguien ha actuado con dolo y, más concretamente, con dolo eventual ${ }^{12}:$ a) En primer lugar, la sentencia determina el elemento cognitivo del dolo afirmando que el acusado "tuvo conocimiento de que el dinero procedía del negocio de las drogas". Tal conclusión se sostiene sobre la base de dos indicios: el hecho de que la cantidad de droga fuera muy importante y la naturaleza "claramente clandestina" de las operaciones; b) Y, en segundo término, la Sala establece también la concurrencia del elemento volitivo del dolo eventual (la asunción o aceptación del origen ilícito del dinero transportado) partiendo para ello de dos indicios: por un lado, del hecho de que el sujeto se hubiera colocado en una situación de ignorancia deliberada acerca de la naturaleza del negocio en el que participaba; y, por otro, de la circunstancia de que se beneficiara económicamente de tal situación.

12 Desde hace varias décadas el Tribunal Supremo se decanta en numerosas resoluciones por el concepto de dolo eventual defendido por las "llamadas teorías de la aceptación". En tal sentido, cfr., por todas, la STS de 10-102006 (ponente Berdugo y Gómez de la Torre). 
Desde luego no cabe ignorar que algunos puntos de esta fundamentación resultan especialmente oscuros, como el que se afirme que el sujeto se encontraba en una situación de ignorancia deliberada respecto del origen del objeto transportado inmediatamente después de haberse sostenido que dicho sujeto "tuvo conocimiento de que el dinero procedía del negocio de las drogas". Ciertamente cabe interpretar que lo que pretende decir la Sala es que el sujeto contaba ya con una sólida sospecha inicial y que su aceptación se deduce del hecho de que no quisiera profundizar o confirmar sus dudas acerca del origen de la cantidad transportada. Sin embargo, tal afirmación no puede realizarse sin presuponer tras la fundamentación de la sentencia argumentos que no aparecen explicitados en su literalidad.

En todo caso, el interés de esta resolución radica en la definición que en ella se ofrece de la ignorancia deliberada como la situación en la que un sujeto no quiere saber aquello que puede y debe conocer, esto es, un estado de ausencia de representación con respecto a un determinado elemento del tipo en el que deben concurrir dos características: la capacidad del sujeto de abandonar dicha situación en caso de haber querido hacerlo y el deber de procurarse dichos conocimientos. A ello se añade un tercer requisito, como es el hecho de que el sujeto se beneficie de la situación de ignorancia por él mismo buscada, sin que la Sala llegue a precisar si debe tratarse de un beneficio económico - como en el caso resuelto- o de cualquier otro tipo.

Con posterioridad, esta doctrina se ha reiterado en resoluciones, como las SSTS de 16-10-2000 o 22-5-2002 (ponente Giménez García), relativa esta última a un caso en el que el acusado alegó desconocer la cantidad exacta de droga que transportaba. Tras estos tres primeros pronunciamientos, ya en el ATS de 4-7-2002 (ponente Martínez Arrieta) se sostuvo que la ignorancia deliberada era "doctrina consolidada de esta Sala" en relación con aquellos casos de acusaciones por tráfico de drogas "en los que el acusado argumenta desconocer el contenido de unos envases que transporta". Según el Tribunal, este planteamiento se basa en la "teoría del asentimiento", "que viene a centrar la esencia del dolo eventual en que el agente si bien desconoce en todos sus detalles el acto ilícito penal en el que se 
encuentra involucrado, lo asume en la medida que acepta todas las consecuencias de su ilícito actuar".

Con todo, si bien la voluntad de no confirmar sus sospechas por parte del sujeto activo era inicialmente considerada por el Tribunal Supremo solo como un indicio del llamado elemento volitivo del dolo eventual ("aceptación", "asentimiento" o "indiferencia"), progresivamente la doctrina de la ignorancia deliberada ha ido cobrando "vida propia", hasta el punto de afirmarse en algunas resoluciones posteriores -de forma más o menos explícita- que en estos casos de provocación del desconocimiento no es siquiera necesario acreditar la concurrencia del elemento cognitivo del dolo para imponer una condena por delito doloso. Así las cosas, el desconocimiento provocado ha alcanzado autonomía como un nuevo título de imputación subjetiva que únicamente se vincula con la figura tradicional del dolo a efectos punitivos. $\mathrm{O}$, si se quiere ver de otra manera, el dolo se ha ampliado a casos que no requieren el elemento cognitivo tal como este último se había perfilado tradicionalmente. En tal sentido cabe citar la STS de 19-1-2005 (ponente Giménez García), en la que, como se desprende del siguiente pasaje, la ignorancia deliberada pasa a convertirse explícitamente en un auténtico sustitutivo del dolo eventual:

"La prueba de conocimiento del delito de referencia es un dato subjetivo, lo que le convierte en un hecho que dada su estructura interna solo podría verificarse -salvo improbable confesión- por prueba indirecta, y en este sentido la constante jurisprudencia de esta Sala ha estimado que a tal conocimiento se puede llegar siempre que se acredite una conexión o proximidad entre el autor y lo que podría calificarse «el mundo de la droga». Esta doctrina se origina en la STS 755/97 de 23 de mayo (...) entre otras, precisándose en la jurisprudencia citada, que no se exige un dolo directo, bastando el eventual o incluso como se hace referencia en la sentencia de instancia, es suficiente situarse en la posición de ignorancia deliberada. Es decir que quien pudiendo y debiendo conocer, la naturaleza del acto o colaboración que se le pide, se mantiene en situación de no querer saber, pero no obstante 
presta su colaboración, se hace acreedor a las consecuencias penales que se deriven de su antijurídico actuar".

En el párrafo reproducido las situaciones de ignorancia deliberada se presentan, de manera explícita, como una forma de imputación subjetiva distinta del dolo directo o el eventual. Ante esta creciente autonomía de esta novedosa forma de imputación subjetiva respecto de las modalidades clásicas de dolo recientemente la Sala Segunda ha intentado delimitar de manera más precisa su contenido y requisitos. En tal sentido conviene destacar de manera especial el esfuerzo de precisión llevado a cabo en la STS de 2-2-2009 (ponente Marchena Gómez), en la que obiter dictum se afirma:

"(...) la experiencia ofrece numerosos ejemplos en los que se producen verdaderas situaciones de ignorancia deliberada. Son casos en los que el autor, pese a colmar todas las exigencias del tipo objetivo, ha incorporado a su estrategia criminal, de una u otra forma, rehuir aquellos conocimientos mínimos indispensables para apreciar, fuera de toda duda, una actuación dolosa, siquiera por la vía del dolo eventual. De esa manera, se logra evitar el tratamiento punitivo que el CP reserva a los delincuentes dolosos, para beneficiarse de una pena inferior -prevista para las infracciones imprudentes-o de la propia impunidad, si no existiera, como sucede en no pocos casos, una modalidad culposa expresamente tipificada. De lo que se trata, en fin, es de fijar los presupuestos que permitan la punición de aquellos casos de ignorancia deliberada en los que se constate la existencia de un acto de indiferencia hacia el bien jurídico que sugiera la misma necesidad de pena que los casos de dolo eventual en su sentido más estricto. Para ello sería necesaria la concurrencia de los siguientes requisitos:

$1^{\circ}$.- Una falta de representación suficiente de todos los elementos que definen el tipo delictivo de que se trate. Esa falta de representación, si es absoluta, nunca podrá fundamentar la imputación subjetiva a título de dolo. Los supuestos abarcados estarán relacionados, de ordinario, con la conciencia de que se va a realizar, con una u otra aportación, un acto inequívo- 
camente ilícito. La sospecha puede incluso no llegar a perfilar la representación de todos y cada uno de los elementos del tipo objetivo, al menos, con la nitidez exigida de ordinario para afirmar la concurrencia del elemento intelectual del dolo. Sin embargo, sí ha de ser reveladora de una grave indiferencia del autor hacia los bienes jurídicos penalmente protegidos, pues, pese a representarse el riesgo que su conducta puede aparejar, no desiste del plan concebido.

$2^{\circ}$.- Una decisión del sujeto de permanecer en la ignorancia, aun hallándose en condiciones de disponer, de forma directa o indirecta, de la información que se pretende evitar. Además, esa determinación de desconocer aquello que puede ser conocido, ha de prolongarse en el tiempo, reforzando así la conclusión acerca de la indiferencia del autor acerca de los bienes jurídicos objeto de tutela penal.

$3^{\circ}$.- Un componente motivacional, inspirado en el propósito de beneficiarse del estado de ignorancia alentado por el propio interesado, eludiendo así la asunción de los riesgos inherentes a una eventual exigencia de responsabilidad criminal" (negrita en el original).

Pese a semejantes pronunciamientos, conviene no pasar por alto que en algunas resoluciones la propia Sala Segunda ocasionalmente ha mostrado reticencias -e incluso auténtico rechazo- ante esta nueva forma de imputación subjetiva. En tal sentido destaca la STS de 20-6-2006 (ponente Bacigalupo Zapater), en la que se afirma, respecto de la propia idea de "ignorancia deliberada", que "tales expresiones no resultan ni idiomática ni conceptualmente adecuadas, dado que si se tiene intención de ignorar es porque, en realidad, se sabe lo que se ignora. Nadie puede tener intención de lo que no sabe. La contradictio in terminis es evidente". Más recientemente estas críticas se han reiterado ocasionalmente en sentencias como las SSTS de 15-2-2011 y 5-4-2011 (ponente Martínez Arrieta), en las que se afirma, respecto a este planteamiento, que "ha sido fuertemente criticado en la doctrina porque se lo entendió como una transposición del 'willful blindness' del derecho norteamericano y porque se considera que no resulta adecuado a las 
exigencias del principio de culpabilidad, cuyo rango constitucional ha puesto de manifiesto el Tribunal Constitucional", 1lamándose asimismo la atención "sobre el riesgo de que la fórmula de la 'ignorancia deliberada' (...) pueda ser utilizada para eludir 'la prueba del conocimiento en el que se basa la aplicación de la figura del dolo eventual', o, para invertir la carga de la prueba sobre este extremo".

\section{IV.2. Posibilidad de un dolo sin conocimiento en la doctrina continental}

La afirmación de que el dolo necesariamente requiere la representación-siquiera como posible- de la concurrencia en un determinado comportamiento de los elementos exigidos por un concreto tipo delictivo apenas se cuestiona en la doctrina continental. El único autor que ha puesto en tela de juicio este auténtico dogma ha sido el alemán Günther Jakobs, quien en algunos de sus trabajos denuncia lo que considera un grave contrasentido de la legislación alemana: el tratamiento dispar existente entre el dolo y el conocimiento de la antijuricidad. Así, mientras en materia de dolo rige el $\S 16 \mathrm{StGB}$, que obliga a castigar a lo sumo como imprudentes aquellos casos en los que falta el conocimiento de las circunstancias del hecho que integran un determinado tipo legal, el $\S 17$ StGB, que se ocupa del error de prohibición, solo establece una atenuación potestativa para el error de prohibición vencible. En otras palabras: mientras en todos los casos de desconocimiento de los elementos del tipo la respuesta ha de ser forzosamente la impunidad o, cuando menos, la importante atenuación que merece la actuación imprudente, pueden existir situaciones de desconocimiento de la antijuricidad que, sin embargo, merezcan la misma pena que los supuestos de auténtico conocimiento ${ }^{13}$.

Para Jakobs este dispar tratamiento resulta difícilmente aceptable, pues obliga a responder de forma injustificadamente benigna ante ciertos supuestos de desconocimiento, concretamente los que denomina casos de "imprudencia dirigida a un fin" o "ceguera ante los hechos"

13 JAKoBs, en Estudios, p. 378. 
(Tatsachenblindheit) ${ }^{14}$. Con todo, si bien la expresión "imprudencia dirigida" parece ser sinónima de ignorancia deliberada, Jakobs no dedica tanto su atención a los casos en que el sujeto se mantiene intencionadamente en el estado de desconocimiento, como a aquellos otros supuestos en los que dicho estado se debe al desinterés, indiferencia o falta de escrúpulos: "por ejemplo" — argumenta este autor_ " "muchas personas desconocen que producen ruidos excesivos o que contaminan el medio ambiente, porque tanto los demás conciudadanos como el medio ambiente les son indiferentes. Si se interesaran por el bien de otros o por el de todos, los efectos perjudiciales les resultarían a todas luces evidentes, pero ese interés falta" ${ }^{15}$.

Para Jakobs resulta inaceptable que estos desconocimientos se beneficien de un tratamiento privilegiado "que no se puede justificar axiológicamente", sino solo "a partir del interés del legislador en establecer una frontera clara y no complicada entre dolo e imprudencia"16. A su juicio resulta un contrasentido que el desconocimiento atribuible a la indiferencia exonere $\mathrm{y}$, en cambio, los conocimientos debidos a un exceso de escrúpulos por parte del sujeto permitan fundamentar una condena por delito doloso ${ }^{17}$. En la misma línea, es muy crítico con regulaciones como la española, que en el art. $14 \mathrm{CP}$ establece la obligación de atenuar siempre que existe un error, sea de tipo o de prohibición, sin importar las causas de la falta de conocimiento ${ }^{18}$.

14 El primer término se utiliza en JAKOBS "Sobre el tratamiento de los defectos volitivos y de los defectos cognitivos", en Estudios, pp. 128-146; el segundo en ID., Derecho penal, pp. 313-314.

15 JaKoBs, en Estudios, p. 138.

16 JaKoBs, en Estudios, p. 139. Ver también ID., Derecho penal, pp. 313-314: "el límite del dolo se determina, al menos en un lugar, rígidamente según hechos psíquicos (conocimiento, desconocimiento), pero no según la valoración de estos hechos".

${ }^{17}$ JAKовs, Derecho penal, p. 313.

${ }^{18}$ Sin embargo, posteriormente, en un trabajo publicado en 2004 el propio JAKOBS, (en Dogmática, pp. 345-358), matiza su planteamiento no descartando la posibilidad de que lege lata el desconocimiento motivado por la indiferencia tenga ya cabida, como un supuesto de dolo, en el texto de la ley. 


\section{Algunas propuestas para un adecuado tratamiento de los casos de ignorancia deliberada}

\section{V.1. Las razones de un distinto tratamiento punitivo}

Para decidir si es correcta la equiparación entre el conocimiento efectivo y la ignorancia buscada parece necesario determinar cuáles son las razones que explican el diferente tratamiento punitivo que tienen previsto en la ley las diversas formas de imputación subjetiva. La cuestión no es pacífica en la doctrina jurídico-penal, habiéndose propuesto diversas explicaciones al respecto ${ }^{19}$. Sin embargo, de todas ellas solo las tres siguientes tratan de establecer vínculos entre la función de la pena y las formas de imputación subjetiva:

a) Por razones de peligrosidad del comportamiento: según esta perspectiva, las realizaciones típicas dolosas son sensiblemente más peligrosas que las imprudentes para los bienes jurídicos que pretende protegerse, lo que explica que exista un mayor interés por parte de los poderes públicos en evitar los primeros comportamientos ${ }^{20}$. Este interés se traduce en la conminación del comportamiento doloso con penas más graves que el meramente descuidado. La principal crítica que se dirige a esta perspectiva consiste en poner de manifiesto cómo, dadas unas mismas circunstancias objetivas, el nivel de riesgo al que se ve sometido un objeto de protección ha de ser, por fuerza, exactamente el mismo con independencia de que el sujeto activo obre de forma dolosa o imprudente. Ciertamente, el autor doloso que persigue un determinado resultado tenderá a modificar su conducta buscando dotar a su actuación de una mayor peligrosidad que asegure las consecuencias pretendidas. Sin embargo, cuando esto suceda el hecho se verá

${ }^{19}$ Para una visión panorámica acerca de esta cuestión cfr. RAGUÉs I VALLĖs, El dolo, pp. 32-42.

${ }^{20}$ Entre los autores que comparten este punto de vista cabe destacar a LuzóN Peña, Curso, p. 331; Mir Puig, El Derecho penal, pp. 69-70 y 103; o Frisch, Vorsatz, p. 100. 
modificado en sus circunstancias externas y, por ello, la comparación en términos estrictamente subjetivos dejará de ser posible ${ }^{21}$.

b) En atención a la peligrosidad personal del sujeto activo, que algunos consideran superior en el caso del delincuente doloso ${ }^{22}$. Desde esta perspectiva, que atiende sobre todo a razones de prevención especial, el reo imprudente se hace acreedor de una pena menos grave porque sus necesidades reeducativas son menores que las del infractor doloso, que ha demostrado con su hecho que se encuentra mucho más alejado de los valores que definen un determinado modelo de sociedad. La objeción que suele formularse a esta perspectiva es que cabe imaginar casos en los que el pronóstico de reincidencia de un delincuente doloso sea muy favorable y no por ello el Derecho en vigor permite tratarle más benignamente que al infractor culposo con un elevado riesgo de reiteración delictiva, ni parece que los ciudadanos estuvieran dispuestos a aceptar un sistema basado en tales premisas

c) Finalmente, una tercera explicación parte del distinto contenido expresivo que se constata en las acciones y omisiones dolosas si se las compara con las imprudentes. Así, mientras en la realización típica el sujeto activo doloso expresa con su hecho la negación de determinados valores compartidos en un determinado modelo social, o de las normas jurídicas que los protegen, en el caso de las realizaciones imprudentes no se advierte tal expresión. Esta diferencia suscita asimismo una distinta necesidad de pena, que también se concibe en términos comunicativos como un acto de reafirmación simbólica por parte del Estado del valor o la norma previamente negados con el hecho delictivo: dado que el delincuente culposo se halla, por lo

${ }^{21}$ Por otra parte, como afirma Feijoo SÁnchez, CPC, 65 (1998), p. 314: "desde el punto de vista del Derecho penal como un mero instrumento maximizador de bienes, la delincuencia imprudente se muestra estadísticamente como más peligrosa y lesiva que la delincuencia dolosa".

${ }^{22}$ Afirma, por ejemplo, HASSEMER, $A D P C P, 1990$, p. 917, que "la reinserción del delincuente doloso supone un 'cambio normativo', una relación transformada del sujeto con la norma, mientras que en el caso del autor de un delito imprudente se trata solo de prestar una atención más elevada o de una previsión de peligro". 
general, expuesto a sufrir una poena naturalis a resultas de su comportamiento, la necesidad de imponerle una pena forense se considera sensiblemente inferior ${ }^{23}$.

Las críticas más habituales a este planteamiento son dos: en primer lugar, se alude a las dificultades con las que se encuentra este modelo explicativo para justificar el castigo de las actuaciones imprudentes, en las que el sujeto no niega explícitamente ninguna norma o valor; y, en segundo término, se mencionan los problemas para explicar por qué la pena, si se concibe como un mero acto declarativo de la vigencia de la norma o el valor negados, debe concretarse en un acto de privación de derechos del reo $^{24}$. La primera objeción se ha tratado de superar apelando a la necesidad de garantizar determinados estándares de peligrosidad, así como al carácter contingente de la pena natural. Y, en relación con la segunda crítica, se ha argumentado que, dado que el delito es una negación objetivada de la norma penal, una pena entendida como reafirmación del ordenamiento negado ha de tener también un determinado contenido objetivo.

Pese a todo, de los tres planteamientos acabados de exponer es este último -el diferente contenido expresivo del dolo y la imprudencia- el que debe acogerse como más acertado pues las dificultades a las que se enfrenta, aun siendo importantes, no parecen insalvables si se introducen los matices oportunos. Así, desde el punto de vista del sistema jurídico-penal todo ciudadano es observado en la doble condición de persona que demanda protección y, al mismo tiempo,

${ }^{23}$ Este planteamiento aparece expuesto, con diversos matrices, en numerosos trabajos de JAKOBS, entre los que cabe mencionar Derecho penal, $\mathrm{p}$. 313; ID., Sociedad, norma y persona, p. 27 ss; y su obra recopilatoria Estudios de Derecho penal, pp. 135-137 y 140-141; p. 116-117; y pp. 371-372 у 378 ss. Más recientemente también Pérez BarberÁ, El dolo, pp. 127-134, fundamenta el tratamiento punitivo propio del dolo en razones de tipo comunicativo. Estos enfoques, basados en planteamientos cercanos a la prevención general positiva, parecen también compatibles con ciertas modalidades de retribución, como el retribucionismo empírico que defienden autores como Robinson, Principios, passim.

${ }^{24}$ Así, entre otros, Schünemann, en Politica criminal, p. 92. 
de potencial infractor ${ }^{25}$. Por tal razón, el mensaje que la pena emite a la ciudadanía también es doble: de un lado, la pena contribuye a la pacificación social dirigiéndose a quienes reclaman su protección mediante la restauración de la vigencia del valor cuestionado por el delito; pero, de otro, la pena envía también a los potenciales infractores el mensaje de que la renuncia a los medios lícitos para conseguir los propios objetivos está condenada al fracaso, buscando así persuadirles de la conveniencia de tratar de alcanzar sus propósitos de acuerdo con la legalidad. Esta última dimensión permite justificar la necesidad de que la pena tenga un contenido aflictivo y no sea una mera desautorización simbólica.

\section{V.2. La ignorancia deliberada: propuesta de tratamiento}

Partiendo de estas premisas, las razones que explican la necesidad de castigar más gravemente los actos dolosos que los imprudentes parecen ser también dos. En primer lugar, para que la pena despliegue su efecto de reafirmación de los valores cuestionados y de pacificación social, su respuesta debe ser mucho más contundente frente a la hostilidad o la grave indiferencia que ante una desconsideración leve, del mismo modo que dicha respuesta también debe ser más o menos intensa en función de la importancia que se otorga al interés lesionado ${ }^{26}$. En se-

25 Para una fundamentación de este planteamiento desde el punto de vista de la teoría de las normas cfr. Silva SÁnchez, en Modernas tendencias, p. 56 ss. La posibilidad de que la prevención general positiva y negativa se ofrezcan como fundamentos simultáneos de la pena se admite por autores como Gimbernat Ordeig, prólogo a Alcácer Guirao, Lesión, p. 16. La evolución en tiempos recientes de cierta parte de la doctrina española "desde una perspectiva intimidatoria de la prevención general hacia otro tipo de perspectiva de influencia más positiva en las consciencias y disposiciones individuales" es descrita por FeIJoo SÁnchez, Retribución, pp. 304-307, con las referencias oportunas.

${ }^{26}$ En este sentido afirma FeiJoo SÁnchez, CPC, 65 (1998), p. 311, que "para el Derecho penal la mayor vinculación del autor con el hecho valorado como injusto hace que el atentado contra la validez de la norma tenga una mayor entidad $y$, consecuentemente, se tenga que aplicar, en principio, la ultima 
gundo término, la pena debe intentar provocar, asimismo, el fracaso del infractor ante la colectividad, lo que exige consecuencias más severas frente a quien ha realizado su propósito destructor del interés protegido (dolo directo de primer grado) y, por tanto, ha alcanzado su objetivo, que ante quien simplemente ha dañado dicho interés como un efecto colateral de su comportamiento (dolo eventual o imprudencia) ${ }^{27}$. En este segundo caso existe además, como señala Jakobs, el riesgo de que el sujeto o sus intereses acaben viéndose dañados por su propio comportamiento (poena naturalis), lo que reduce o incluso puede eliminar la necesidad de provocar con la pena forense el fracaso del delincuente. Por el contrario, tal necesidad subsiste plenamente cuando el éxito del sujeto pasaba precisamente por la destrucción del interés tutelado.

Partiendo de estas premisas cabe plantearse cuál debe ser el tratamiento de los casos de representación del riesgo en los que no concurre intención de menoscabar el interés protegido. Desde un punto de vista expresivo, tanto en los casos de mero conocimiento como de desconocimiento se advierte una indiferencia hacia el valor lesionado: en el primer supuesto porque el sujeto no desiste de sus planes pese a ser consciente de que estos entrañan un riesgo de lesión; y en el segundo porque el interés lesionado importa tan poco al sujeto que este ni tan siquiera se da cuenta de que lo pone en peligro. Con todo, entre ambas situaciones existe una distinción que radica en el hecho de que mientras en el caso del conocimiento la indiferencia tiene como referente concreto el objeto de protección, en el caso de desconocimiento tal indiferencia se manifiesta, de manera más difusa, respecto de todos aquellos intereses

ratio de forma más contundente para recuperar la confianza en la norma que se ha visto perturbada". También SchünEMAnN, en Temas actuales, p. 105 , concede cierta importancia al elemento expresivo de la pena del dolo (con la función de "establecer simbólicamente tabúes mediante la pena más grave"), si bien entremezclada con el argumento de la mayor peligrosidad del comportamiento doloso para los bienes jurídicos.

${ }^{27}$ Esta dimensión del castigo ha sido introducida en tiempos recientes en el planteamiento de Jakobs, como destacan CAncio Melí́/FeiJoo SÁnchez, en JAKoBs, La Pena Estatal, p. 57 ss. Para los argumentos del propio autor, cfr. ibidem, p. 142. 
susceptibles de ser lesionados. Esta sutil diferencia puede explicar que se traten de manera distinta el dolo eventual y la imprudencia.

Los casos de ignorancia deliberada stricto sensu parecen situarse en una situación intermedia, especialmente cuando el sujeto ha partido de la sospecha inicial de que podía lesionar algún interés indeterminado pero tal sospecha no ha resultado ser para él un motivo suficiente para confirmar la existencia de tal riesgo y, llegado el caso, evitar la conducta lesiva. Por otra parte, a efectos de valorar en estos casos la necesidad de sanción parece indispensable tener en cuenta también las razones que han llevado al sujeto a no querer profundizar en sus sospechas. En tal sentido conviene diferenciar entre los casos de ignorancia deliberada atribuible a debilidad moral de aquellos casos en los que el individuo ha buscado beneficiarse de su estado de ignorancia para eludir responsabilidades. En un original trabajo publicado en 1999, el profesor estadounidense David Luban diferencia entre dos modelos de ignorantes deliberados: por un lado, el que puede ser visto como un avestruz, esto es, como un débil moral que trata de negarse a sí mismo el hecho de que se enfrenta a un dilema de naturaleza también moral, en cuyo caso su actitud parece menos grave que la actuación con conocimiento cierto sobre los datos relevantes de la propia conducta; y, por otro lado, el que sujeto merece ser equiparado a un astuto zorro, es decir, alguien que ha optado decididamente por llevar a cabo una conducta ilícita y que ha buscado la propia ignorancia solo como una excusa para protegerse frente a posibles declaraciones de culpabilidad. En este caso, sostiene Luban, la ignorancia podría considerarse incluso como más grave que el mero conocimiento, pues añade a la consciencia un elemento de cálculo obstinado que la hace más culpable ${ }^{28}$.

En estos últimos supuestos se aprecia una necesidad de pena superior a la de los casos normales de negligencia por dos grandes razones. En primer lugar, porque desde un punto de vista expresivo no solo cabe valorar el desconocimiento, sino los motivos que han llevado a él: no en vano, un observador medio nunca diría que quien ha querido mantenerse en la ignorancia se encuentra en una situación de error, máxime si partía 
de la sospecha inicial de que su comportamiento podía lesionar algún interés -ciertamente no concretado-colectivo o de otro sujeto. Y, en segundo término, porque si semejantes casos quedan en la impunidad, la pena estará dejando de cumplir su función de provocar el fracaso del delincuente en situaciones en las que, sin embargo, este habrá alcanzado su propósito criminal. Con la impunidad en tales casos se dirigiría a la ciudadanía el mensaje de que en determinadas circunstancias peligrosas para terceros resulta más aconsejable inhibirse que preocuparse. En resumen: en aquellos casos en los que el sujeto ha contado con una sospecha inicial de que su conducta presente o futura podía resultar lesiva para algún interés penalmente relevante pero ha preferido mantenerse en un estado de ignorancia como una estrategia para, llegado el caso, poder alegar dicha ignorancia en su descargo cabe advertir una necesidad de sanción similar a la de los casos de dolo eventual y, desde luego, superior a los supuestos habituales de negligencia.

Cómo deba trasladarse esta distinta valoración a la lex lata es ya una cuestión de cada sistema jurídico. En ordenamientos como el argentino o el español no parece que existan problemas insalvables de legalidad para incluir estos supuestos en el concepto de dolo, una expresión muy abierta, de carácter eminentemente técnico y de la que no existe definición legal, aunque conviene tener presente que la inclusión de semejantes casos en el concepto de dolo impedirá seguir definiendo esta figura a partir del conocimiento. Cuestión distinta, aunque más compleja, es el concreto tratamiento penológico que esta modalidad de dolo, si se acepta, deberá merecer en los sistemas continentales, en los que la gradación de las consecuencias jurídicas por razones de imputación subjetiva -la distinción simplista entre dolo y culpa- no es lo bastante rica como para captar la distinta valoración que merecen las diferentes situaciones que se dan en la práctica.

Las anteriores conclusiones y, en particular, los problemas para obtener una definición generalmente válida de dolo, deberían ser un motivo para reflexionar acerca de la necesidad de replantear un sistema de imputación subjetiva basado exclusivamente en la distinción entre dolo e imprudencia o, cuando menos, para revisar las consecuencia jurídicas que se asocian a cada una de estas figuras. El hecho de que bajo 
un único concepto de dolo -y, en principio, con la misma respuesta punitiva- deban incluirse situaciones tan diversas entre sí como la intención, la mera representación del riesgo o ciertos casos de desconocimiento provocado permite afirmar que esta figura no solo está conceptualmente "sobrecargada", sino que, además, su aplicación plantea importantes problemas de proporcionalidad ${ }^{29}$. En palabras de Schünemann, resulta dudoso que la tradicional distinción entre dolo y culpa pueda "realmente soportar y legitimar aquellas enormes diferencias en las consecuencia jurídicas que existen entre el delito doloso y el culposo, tanto en relación con el alcance de la responsabilidad penal, como así también respecto de la cuantía de la amenaza penal"30.

En tales circunstancias de lege ferenda parece imponerse una evolución hacia un sistema que -en la línea del citado Código Penal $M_{o d e l o}{ }^{31}$ - acoja un mayor número de modalidades de imputación subjetiva que permitan captar adecuadamente las peculiaridades de cada caso y otorgar un tratamiento más proporcionado en las consecuencias. Entre estas situaciones particulares que merecen un tratamiento específico que no puede consistir en una reconducción automática a los casos de intención, conocimiento o desconocimiento se cuentan, sin duda, los supuestos de ignorancia deliberada en sentido estricto que se han descrito en las anteriores páginas. Estos casos son, como se anunciaba, una pequeña brecha que pone en evidencia algunos de los graves problemas que presenta el vigente sistema continental de imputación subjetiva.

${ }^{29}$ Expone sólidas razones para cuestionar la equiparación entre las consecuencias de todas las formas de dolo Molina Fernández, en Rodríguez Mourullo- $L H$, p. 739, proponiendo introducir "un sistema categorial más rico, que pueda dar cuenta de la progresividad", como se hace en la imprudencia, en la que distingue entre grave y leve. Interesantes al respecto también las consideraciones de MANRIQue Pérez, Acción, pp. 274-275, partiendo de la doctrina del doble efecto.

${ }^{30}$ Schünemann, en Temas actuales, p. 100.

${ }^{31} \mathrm{Al}$ respecto cfr. el estudio comparado de TAYLOR, $R J, 17$ (2004) p. 374. 
Mejor no saber

\section{Bibliografía}

Alcácer Guirao, Rafael, ¿Lesión de bien jurídico o lesión de deber? Apuntes sobre el concepto material de delito, Barcelona, 2003.

Charlow, Robin, "Wilful Ignorance and Criminal Culpability", Texas Law Review, 70 (1992), pp. 1351-1429.

FeIJoo SÁnchez, Bernardo, "La distinción entre dolo e imprudencia en los delitos de resultado lesivo. Sobre la normativización del dolo", Cuadernos de Política Criminal, 65 (1998), pp. 269-364.

FEIJOO SÁNCHEZ, Bernardo, Retribución y prevención general. Un estudio sobre la teoría de la pena y las funciones del Derecho penal, Montevideo, 2007.

Frisch, Wolfgang, Vorsatz und Risiko, Colonia, Berlín, Bonn, Múnich, 1983.

Hassemer, Winfried, "Los elementos característicos del dolo", trad. M.M. Díaz Pita, Anuario de Derecho Penal y Ciencias Penales, 1990, p. 909 ss.

Husak, Douglas N. / Callender, Craig A., "Wilful Ignorance, Knowledge, and the 'Equal Culpability' Thesis: a Study of the Deeper Significance of the Principle of Legality", Wisconsin Law Review, 29 (1994), pp. 29-69.

IoWA LAW REVIEW (COMMENTs), "Willful Blindness as a Substitute for Criminal Knowledge”, ID, 63 (1977), pp. 466-485.

JakoBs, Günther, Derecho penal. Parte general. Fundamentos y teoría de la imputación, 2. ${ }^{a}$ ed., trad. J. Cuello Contreras y J.L. Serrano González de Murillo, Madrid, 1995.

JAKOBS, Günther, Sociedad, norma y persona en una teoría de un Derecho penal funcional, trad. M. Cancio Meliá y B. Feijoo Sánchez, Madrid, 1996.

JAKOBS, Günther, trad. Suárez González, Peñaranda Ramos y Cancio Meliá, Estudios de Derecho penal, Madrid, 1997.

JAKoBs, Günther, "Indiferencia como dolo indirecto", trad. C. Pérez del Valle, en López BARJa de Quiroga / Zugaldía Espinar (coords.), Dogmática y ley penal. Libro homenaje a Enrique Bacigalupo, tomo I, Madrid-Barcelona, 2004, pp. 345-358.

JAKoBs, Günther, La Pena Estatal: Significado y Finalidad, trad. M. Cancio Meliá y B. Feijoo Sánchez, Cizur Menor, 2006. 
LubAn, David, "Contrived Ignorance", The Georgetown Law Journal, 87 (1999), pp. 957-980.

Luzón Peña, Diego-Manuel, Curso de Derecho penal. Parte general I, Madrid, 1996.

ManriQue Pérez, María Laura, Acción, dolo eventual y doble efecto, Madrid, Barcelona, Buenos Aires, 2012.

MArcus, Jonathan L., "Model Penal Code Section 2.02(7) and Willful Blindness", The Yale Law Journal, 102 (1993), pp. 2231-2257.

Mir Puig, Santiago, El Derecho penal en el Estado social y democrático de Derecho, Barcelona, 1994.

Molina Fernández, Fernando, "La cuadratura del dolo: problemas irresolubles, sorites y Derecho penal", en VVAA, Homenaje al profesor Dr. Gonzalo Rodríguez Mourullo, Madrid, 2005, pp. 691-742.

Pérez BarberÁ, Gabriel, El dolo eventual. Hacia el abandono de la idea de dolo como estado mental, Buenos Aires, 2011.

Ragués i Vallès, Ramon, El dolo y su prueba en el proceso penal, Barcelona, 1999.

Ragués I Vallès, Ramon, La ignorancia deliberada en Derecho penal, Barcelona, 2007.

RobBIns, Ira P., "The Ostrich Instruction: Deliberate Ignorance as a Criminal Mens Rea", The Journal of Criminal Law and Criminology, 81 (1990), pp. 191-234.

Robinson, Paul H., Principios distributivos del Derecho penal, trad. M. Cancio Meliá e I. Ortiz de Urbina Gimeno, Madrid, Barcelona, Buenos Aires, 2012.

SchüNEMANN, Bernd, "Die deutschsprachige Strafrechtswissenschaft nach der Strafrechtsreform im Spiegel des Leipziger Kommentars und des Wiener Kommentars" (1. " parte), Goltdammer's Archiv für Strafrecht, 1985, p. 341 ss.

SchünEMANN, Bernd, "Sobre la crítica a la teoría de la prevención general positiva", trad. P. Sánchez-Ostiz Gutiérrez, en Silva SÁnchez (ed.), Politica criminal y nuevo Derecho Penal. Libro Homenaje a Claus Roxin, Barcelona, 1997, pp. 89-100.

Schünemann, Bernd, "De un concepto filológico a un concepto tipológico de dolo”, trad. M. Sacher y C. Suárez González, en ID., 
Temas actuales y permanentes del Derecho penal después del milenio, Madrid, 2002, pp. 97-111.

Silva SÁnchez, Jesús-María, “¿Directivas de conducta o expectativas institucionalizadas? Aspectos de la discusión actual sobre la teoría de las normas", en VVAA, Modernas tendencias en la Ciencia del Derecho Penal y en la Criminología, Madrid, 2002, pp. 559-575.

TAYLOR, Greg, "The Intention Debate in German Criminal Law", Ratio Juris, 17 (2004), pp. 346-380. 